\title{
The pitfalls of empirical fitting of glass relaxation data with stretched exponents
}

\author{
M. Atzmon ${ }^{\text {a) }}$ \\ Departments of Nuclear Engineering and Radiological Sciences and Materials Science and Engineering, \\ University of Michigan, Ann Arbor, Michigan 48109-2104, USA
}

(Received 29 September 2017; accepted 20 January 2018; published online 9 February 2018)

\begin{abstract}
A stretched exponent is commonly used to fit experimental relaxation data for glasses, which typically exhibit a range of time constants. While it has been supported by theory for various processes, mostly near and above the glass transition temperature, $T_{g}$, it is also commonly used in phenomenological fits below $T_{g}$ without a mechanistic model. The properties of the stretched exponent, and sample data for the enthalpy release and dynamic-mechanical response of metallic glasses, are used to show that in the absence of a mechanistic model, stretched-exponent fits easily lead to artifacts and flawed conclusions. Published by AIP Publishing. https://doi.org/10.1063/1.5007056
\end{abstract}

Processes with an exponential time dependence are ubiquitous in physical, chemical, and biological systems. Examples include the decay of radioactive isotopes, chemical species or heat, and damped mechanical or electrical systems. Such processes, for which the rate of change of a measured quantity is proportional to the quantity itself, behave as $\exp (-\lambda t)$, where $\lambda$ is a decay constant. The characteristic time constant, $\tau=1 / \lambda$, for a decrease by a factor of $\exp (-1)$ is the same over any time interval. Exponential behavior with a distribution of time constants often exists, e.g., for diffusion or mechanical relaxation in amorphous solids. ${ }^{1-5}$ There is no explicit expression that yields the distribution from the measured time dependence, but this "inverse problem" may be solved by using least-squares fits if the signal-to-noise ratio is sufficiently high. ${ }^{3-7}$

Exponential relaxation processes with a distribution of time constants are measured in a wide range of glasses by various means, including mechanical or dielectric or correlation spectroscopy with neutrons, photons, or electrons. Such data are often analyzed by least-squares fitting an empirical expression for the time dependence-a stretched exponent ${ }^{8-10}$

$$
x(t)=x(0) \exp \left(-\left(t / \tau_{s}\right)^{\beta}\right),
$$

where $\tau_{\mathrm{s}}$ and $\beta$ are constants and $\beta$ is related to the width of the distribution. Fits of Eq. (1) require three parameters, $x(0), \tau_{\mathrm{s}}$, and $\beta$. Its use implies an a priori assumption about the functional form of the distribution of time constants. First used to describe the discharge of a capacitor, ${ }^{8}$ the expression is also referred to as Kohlrausch-Williams-Watts $(\mathrm{KWW}) .{ }^{9,10}$ A corresponding approximate analytical expression has been used to describe the measurements in the frequency domain. ${ }^{11}$ In keeping with convention, both the stretched exponent and its frequency-domain expression will also be referred to as KWW. Mechanistic models have been derived which support the use of Eq. (1) for specific processes, mostly near or above the glass transition temperature, $T_{g}{ }^{12-15}$ In Ref. 12 , the theory is shown to predict $\beta$

\footnotetext{
a)Authors to whom correspondence should be addressed: atzmon@umich.edu
}

accurately for several phenomena if extrinsic effects are accounted for. In Ref. 14, the common assumption of a temperature-independent $\beta$ for fast ionic conductors is disputed. $\beta$ is shown to behave as $T^{0.5}$ for a fixed distribution of activation energies, in agreement with experiment. In Ref. 15, it is shown that $\beta$ only provides a lower limit on the fragility of a supercooled liquid. Reference 16 compares the analysis of dielectric loss spectra of organic glasses in terms of a physical model with that using empirical expressions for the distribution of time constants. Reference 17 compares empirical expressions for the distribution and discusses the associated ambiguities. There is a large body of literature in which Eq. (1) is used empirically without a mechanistic model, e.g., Refs. 18-26. Such a use is motivated by the fact that it typically yields good fits with only three fitting parameters.

In this paper, focusing on temperatures well below $T_{g}$, the lack of time-shift invariance of KWW is first discussed, which is shown to potentially yield inconsistent results. Published enthalpy-release data for a relaxing metallic glass are shown to be described better with bimolecular kinetics than with the originally used KWW expression. KWW fits are performed to loss moduli computed from quasi-static anelastic relaxation data that are described well with a single mechanistic model. A fit discrepancy at high frequencies is shown to incorrectly imply_according to an oft-used line of reasoning - two distinct mechanisms.

The lack of time-shift invariance of Eq. (1) is obvious on analytical grounds and is demonstrated and discussed below. This is significant since some experimental techniques preclude an exact determination of the initial time. For example, for enthalpy-release measurements by isothermal calorimetry, a finite time is required for raising the temperature from ambient. Heat release during this time also leads to an uncertainty in the initial state. Both effects will increase with increasing temperature, thus producing an artifact in the temperature dependence of the fitting parameters. In Fig. 1, a hypothetical time-dependent quantity, described as a stretched exponent with a small shift that simulates an uncertainty in initial time, $y(t)=\exp \left(-((t+3) / 30)^{0.5}\right)$, is fitted with an unshifted stretched exponent [Eq. (1)]. $x(0), \tau_{\mathrm{s}}$, and $\beta$ 


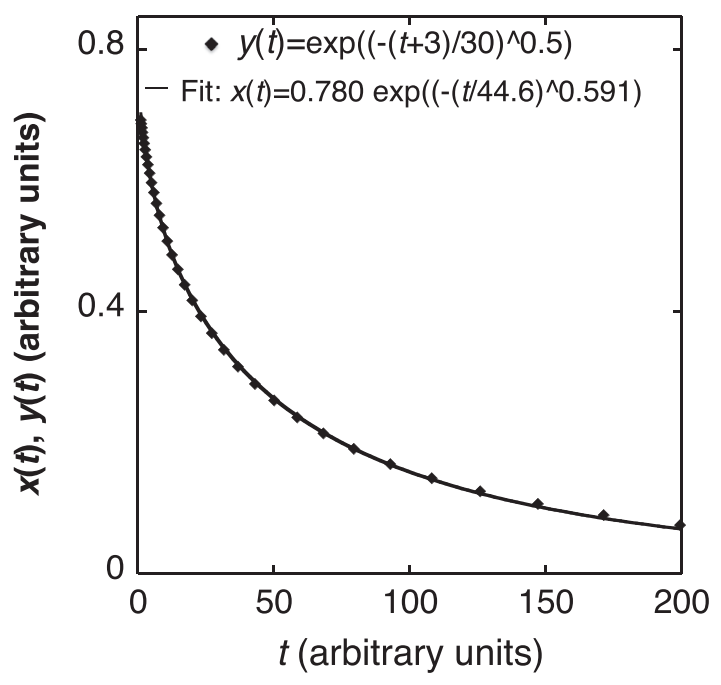

FIG. 1. A hypothetical time-dependent quantity, described as a stretched exponent with a small shift [y(t), diamonds], is fitted with an unshifted stretched exponent $[x(t)$, Eq. (1), line]. Significantly different $\tau$ and $\beta$ are obtained.

are the fitting parameters. An excellent fit is obtained, with significantly different parameters, $\beta=0.591$ and $\tau=44.6$. The first data point in Fig. 1 is at $t=1$, and using different values results in the variation of the fitting parameters. These results provide a powerful example of the closeness of fit provided by a stretched exponent to a function that is not in the form of Eq. (1) while yielding inconsistent fitting parameters. It follows that also for any data fitted with KWW, if the initial time $t=0$ is uncertain, the fitting parameters will not yield meaningful information. In contrast, for a spectrum of exponential relaxations obtained from time-dependent data, a time shift in the latter would only lead to a change in amplitude for small time constants, leaving the general spectrum features unchanged.

The result of Fig. 1 is a manifestation of the fact that, unlike exponential behavior, for which the characteristic time constant is the same for any $t$, the relative change rate for the stretched exponent evolves in time: its instantaneous rate constant, $-\dot{x}(t) / x(t)=(\beta / \tau)(t / \tau)^{\beta-1}$, where $\dot{x}(t)$ is the temporal derivative, is singular at $t=0$ for $0 \leq \beta<1$ and decreases with time. Many studies consider $\tau$ to be an average time constant, but for a systematic variation of $\beta$, e.g., with temperature, $\Gamma(1+1 / \beta) \tau$, an average over $0 \leq t \leq \infty$ has been used. ${ }^{27} \Gamma$ is the gamma function. For measurements of thermally activated processes with a distribution of activation free energies, even the use of this average is problematic: if the ranges of $t / \tau$ probed are small and vary with temperature, ${ }^{28,29}$ anomalies in the temperature dependence of $\tau$ and $\beta$ may result. For example, for exponential relaxations with a distribution of activation energies, the active part of the latter will become narrower with decreasing temperature, resulting in $\beta$ approaching 1 . This is particularly relevant for sluggish atomic transport, e.g., mechanical relaxation, well below $T_{g}$.

Glasses relax continuously toward a temperaturedependent metastable equilibrium, a process conveniently monitored by the calorimetric measurement of the enthalpy evolution. Thus-acquired data have often been analyzed using KWW fits, e.g., Refs. 16-20. The same has been done directly for the associated free-volume annihilation process. ${ }^{30}$ Since relaxation has been successfully modeled with bimolecular kinetics, ${ }^{31-33}$ a corresponding fit will next be discussed and compared with KWW. The term bimolecular kinetics refers to the rate at which two molecules, or species in general, react to form a new molecule or defect. This rate is proportional to the product of concentrations of the species involved or to the square of the concentration when identical members of a species react with each other. For illustration, when vacancies and interstitials are formed by irradiation of a crystalline solid, the rate at which they recombine is described by bimolecular kinetics. Under a rate of vacancyinterstitial pair production of $K_{0}$, the concentrations of vacancies and interstitials, $c_{v}$ and $c_{i}$, are given, respectively, by the solutions to ${ }^{34}$

$$
\frac{d c_{v}(t)}{d t}=K_{0}-K_{v} D_{v} c_{v}-\alpha c_{i} c_{v}
$$

and

$$
\frac{d c_{i}(t)}{d t}=K_{0}-K_{i} D_{i} c_{i}-\alpha c_{i} c_{v}
$$

where $K_{d}$ and $D_{d}(d=i$ or $v)$ are the strengths of sinks and diffusion coefficients, respectively, for the respective defects, and the recombination constant $\alpha$ is proportional to $D_{i}+D_{v}$ $\left(\approx D_{i}\right)$. Dislocations and grain boundaries, for example, act as sinks for point defects. Thermal equilibrium concentrations are neglected here. Under irradiation, $K_{O}>0, c_{i}$ and $c_{v}$ reach the steady state when the rate of defect removal equals that of defect production. When the irradiation is turned off, $c_{i}$ and $c_{v}$ decay to zero. In a special case for which recombination is negligible, $c_{i}(t)$ and $c_{v}(t)$ evolve exponentially, with characteristic time constants of $\left(K_{i} D_{i}\right)^{-1}$ and $\left(K_{v} D_{v}\right)^{-1}$, respectively. When sinks are negligible, $c_{v}=c_{i} \equiv c$. If $K_{O}$ is turned off at $t=0, c(t)$ evolves as

$$
c_{b}(t)=\frac{c(0)}{\alpha c(0) t+1} .
$$

While an effective half-life $\tau_{1 / 2}=1 /(\alpha c(0))$ can be defined, it is obviously not characteristic of the process since it is dependent on the initial value $c(0)$. As the system evolves, $c_{b}(t)$ decreases and the subsequent $\tau_{1 / 2}$ increases as a result. Therefore, bimolecular kinetics do not have a characteristic time constant-the instantaneous rate constant evolves as $-\dot{c}_{b}(t) / c_{b}(t)=\alpha c(0) /(\alpha c(0) t+1)$. Although defects in amorphous solids are not as well defined as in crystals, viscosity and diffusion data have been interpreted in terms of bimolecular annealing kinetics of dangling bonds in amorphous $\mathrm{Si}$ and $\mathrm{Ge}^{31,32}$ and of free volume in metallic glasses. $^{33}$

As a first example, sample enthalpy relaxation data will be analyzed with the bimolecular model, Eq. (3), and the result compared with the originally used KWW fit. Figure 2 shows the enthalpy release of a metallic glass as a function 


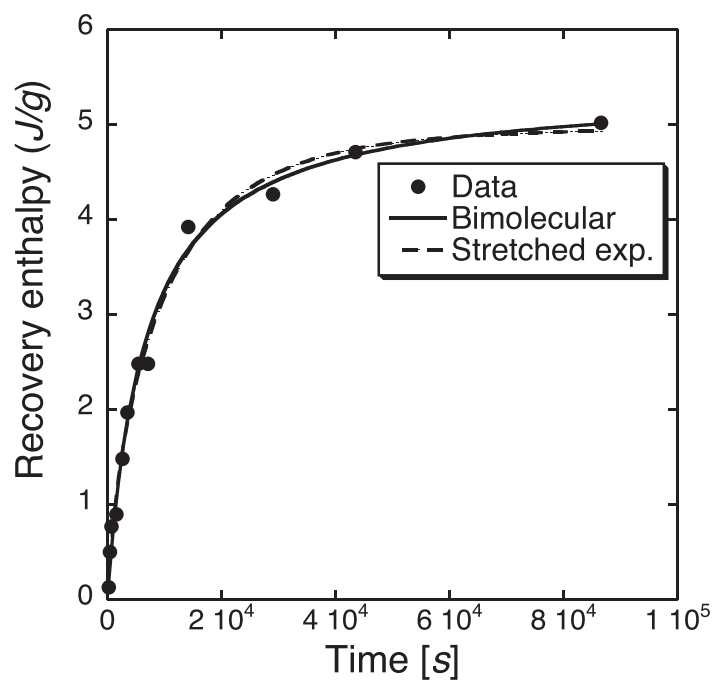

FIG. 2. Enthalpy recovery data of Ref. 19, fitted using both Eqs. (1) and (4). The former yields $\tau_{s}=9555 \mathrm{~s}$ and the latter $\tau_{1 / 2}=6551 \mathrm{~s}$

of annealing time, ${ }^{19}$ originally fitted with KWW. Following Ref. 33, the process is presently attributed to free-volume annihilation with bimolecular kinetics. Assuming that the enthalpy change is dominated by the free volume and proportional to it, we obtain

$$
\Delta H \propto \Delta H_{\infty}\left(1-\frac{c_{b}(t)}{c(0)}\right)=\Delta H_{\infty}\left(\frac{\alpha c(0) t}{\alpha c(0) t+1}\right),
$$

where $\Delta H_{\infty}$ is the value of $\Delta H$ for $t \rightarrow \infty . c(t)$ here represents the amount of free volume. Fits based on both Eqs. (1) and (4) are shown in Fig. 2. The corresponding values of the goodness-of-fit parameter, $\chi^{2}, 35$ i.e., the sum of the normalized squares of differences between the data points and the fit is 0.290 and 0.307 , respectively. The values of reduced $\chi^{2}$, i.e., $\chi^{2}$ divided by the number of degrees of freedom in the fit, ${ }^{35}$ are 0.0322 and 0.0307 . The number of degrees of freedom is the number of data points minus that of the fitting parameters. Thus, three fitting parameters in Eq. (1) vs. two in Eq. (4) $\left[\Delta H_{\infty}\right.$ and $\left.\alpha c(0)\right]$ do not improve the fit quality. Moreover, the bimolecular fit relies on a physical model, whereas KWW does not provide mechanistic information in this case. Equation (4) can be similarly applied to other studies, e.g., Refs. 18 and 20-22.

Next, an empirical KWW fit to data in the frequency domain $^{11}$ is examined. Such data are obtained with cyclic stimulation, e.g., mechanical or dielectric. ${ }^{36}$ Discrepancies between the loss modulus or dielectric loss and their empirical fits are commonly used to invoke separate mechanisms, e.g., Refs. 23-26. It will now be shown that such a discrepancy, in and of itself, is insufficient for asserting the existence of a second process. For this purpose, our quasi-static anelastic relaxation data for amorphous $\mathrm{Al}_{86.8} \mathrm{Ni}_{3.7} \mathrm{Y}_{9.5}$, obtained over eight orders of magnitude in time, are used., 47 A detailed spectrum of time constants has been obtained and analyzed quantitatively with a single mechanism - an atomically quantized hierarchy of shear transformation zones (STZs).

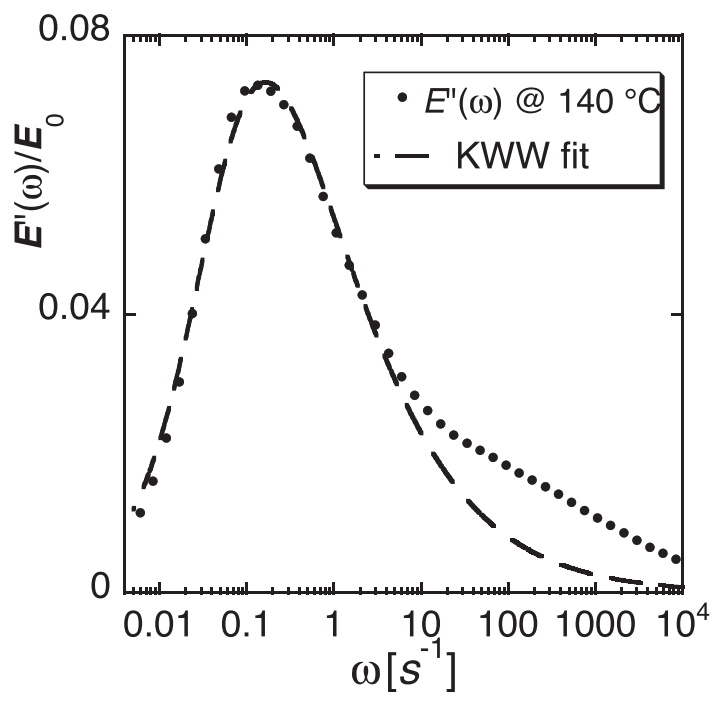

FIG. 3. Loss modulus of amorphous $\mathrm{Al}_{86.8} \mathrm{Ni}_{3.7} \mathrm{Y}_{9.5}$ at $413 \mathrm{~K}$ as a function of frequency, normalized by the high-frequency modulus, calculated from quasi-static data. ${ }^{4}$ See Ref. 38 for details.

In Fig. 3, the loss modulus of $\mathrm{Al}_{86.8} \mathrm{Ni}_{3.7} \mathrm{Y}_{9.5}, E^{\prime \prime}$, at $413 \mathrm{~K}$ is, shown as a function of frequency, as calculated ${ }^{38}$ based on the spectrum of Ref. 4 and the associated model. An analytical KWW approximation ${ }^{11}$ fits $E^{\prime \prime}$ well for most of the frequency range but deviates from its high-frequency tail. Since $E^{\prime \prime}$ was calculated from the data on the basis of a single mechanism, it is obvious that this deviation does not imply the existence of a separate mechanism. The tail, often termed "excess wing," is in the present case simply due to small STZs, which have a lower activation free energy and therefore a shorter time constant. Obviously, there is a physical reason to invoke two distinct relaxation processes in nonrigid molecular glasses, for example. This is less obvious in metallic glasses, unless a distinct high-frequency peak is observed. ${ }^{39}$ The latter case may be explained with local composition effects underlying distinct high-frequency relaxation events, e.g., Refs. 40 and 41.

The present results illustrate the limitations of KWW and more generally of phenomenological fits. One consequence is that for measurements with uncertain $t=0$, because of the measurement-dependent systematic variation of the KWW fitting parameters with temperature discussed earlier, activation free energies based on $\tau$ will be unreliable. The main message of this paper is not to propose a specific model. Rather, it is to highlight the importance of using a mechanistic model when fitting experimental data whenever possible, instead of KWW or other phenomenological expressions. For example, if the underlying process is believed to result from simple exponential relaxation with a distribution of time constants, and the data scatter is small, an attempt should be made to compute the spectrum of relaxation times from the data. In Ref. 5, for example, the temperature dependence of each peak in the spectrum was obtained. Bimolecular annihilation kinetics, e.g., of free volume, may be relevant in other cases.

In summary, when analyzing relaxation data, which do not obey simple exponential kinetics, fitting parameters 
obtained from phenomenological KWW fits without a mechanistic model may suffer from significant artifacts and even lead to false qualitative conclusions. Great caution should be exercised when assigning a physical meaning to such parameters, and mechanistic models should be explored whenever possible. This general conclusion is likely to apply to a broad range of materials and phenomena.

This work was funded by the U.S. National Science Foundation (NSF), Grants Nos. DMR-1307884 and DMR1708043. The author thanks Professor F. Spaepen for useful discussions.

${ }^{1}$ M. Atzmon and F. Spaepen, Science and Technology of Rapidly Quenched Alloys, edited by M. Tenhover, W. L. Johnson, and L. E. Tanner (Mater. Res. Soc. Symp. Proc., 1987), Vol. 80, pp. 55-59.

${ }^{2}$ A. S. Argon and H. Y. Kuo, J. Non-Cryst. Solids 37, 241 (1980).

${ }^{3}$ J. R. Cost, J. Appl. Phys. 54, 2137 (1983).

${ }^{4}$ J. D. Ju, D. Jang, A. Nwankpa, and M. Atzmon, J. Appl. Phys. 109, $053522(2011)$

${ }^{5}$ J. D. Ju and M. Atzmon, Acta Mater. 74, 183 (2014)

${ }^{6} \mathrm{~J}$. Honerkamp and J. Weese, Macromolecules 22, 4372 (1989).

${ }^{7}$ H. Schäfer, U. Albrecht, and R. Richert, Chem. Phys. 182, 53 (1994).

${ }^{8}$ R. Kohlrausch, Ann. Phys. Leipzig 91, 56 (1854).

${ }^{9}$ G. William and D. C. Watts, Trans. Faraday Soc. 66, 80 (1970)

${ }^{10}$ G. Williams, D. C. Watts, S. B. Dev, and A. M. North, Trans. Faraday Soc. 67, 1323 (1971).

${ }^{11}$ R. Bergman, J. Appl. Phys. 88, 1356 (2000).

${ }^{12}$ J. C. Phillips, Rep. Prog. Phys. 59, 1133 (1996).

${ }^{13}$ J. R. MacDonald, J. Appl. Phys. 62, R51 (1987)

${ }^{14}$ I. Svare, S. W. Martin, and F. Borsa, Phys. Rev. B 61, 228 (2000).

${ }^{15}$ P. K. Gupta and J. C. Mauro, Phys. Rev. E 78, 062501 (2008).

${ }^{16}$ C. Hansen, R. Richert, and E. W. Fisher, J. Non-Cryst. Solids 215, 293 (1997).

${ }^{17}$ C. P. Lindsey and G. D. Patterson, J. Chem. Phys. 73, 3348 (1980).

${ }^{18}$ I. M. Hodge, J. Non-Cryst. Solids 169, 211 (1994), and references therein

${ }^{19}$ J. C. Qiao and J. M. Pelletier, Intermetallics 19, 9 (2011).
${ }^{20}$ R. Raghavan, P. Murali, and U. Ramamurty, Metall. Mater. Trans. A 39, 1573 (2008).

${ }^{21}$ K. Kawai, T. Hagiwara, R. Takai, and T. Suzuki, Pharm. Res. 22, 490 (2005), and references therein.

${ }^{22}$ L. Hu and Y. Yue, J. Phys. Chem. C 113, 15001 (2009).

${ }^{23}$ J. C. Qiao, R. Casalini, and J. M. Pelletier, J. Non-Cryst. Solids 407, 106 (2015).

${ }^{24}$ Z. F. Zhao, P. Wen, C. H. Shek, and W. H. Wang, Phys. Rev. B 75, 174201 (2007).

${ }^{25}$ P. Rösner, K. Samwer, and P. Lunkenheimer, Europhys. Lett. 68, 226 (2004).

${ }^{26}$ R. Brand, P. Lunkenheimer, U. Schneider, and A. Loidl, Phys. Rev. Lett. 82, 1951 (1999).

${ }^{27}$ M. N. Berberan-Santos, E. N. Bodunov, and B. Valeur, Chem. Phys. 315, 171 (2005).

${ }^{28}$ A. Belardini, L. Dominici, M. C. Larciprete, and F. Michelotti, Appl. Phys. Lett. 89, 231110 (2006), Figs. 3 and 4.

${ }^{29}$ J. C. Qiao, Y.-J. Wang, L. Z. Zhao, L. H. Dai, D. Crespo, J. M. Pelletier, L. M. Keer, and Y. Yao, Phys. Rev. B 94, 104203 (2016), Fig. 1.

${ }^{30}$ A. Ishii, F. Hori, A. Iwase, and Y. Fukumoto, Mater. Trans. 49, 1975 (2008).

${ }^{31}$ S. Roorda, W. C. Sinke, J. M. Poate, D. C. Jacobson, S. Dierker, B. S. Dennis, D. J. Eaglesham, F. Spaepen, and P. Fuoss, Phys. Rev. B 44, 3702 (1991).

${ }^{32}$ A. Witvrouw and F. Spaepen, J. Appl. Phys. 74, 7154 (1993).

${ }^{33}$ S. S. Tsao and F. Spaepen, Acta Metall. 33, 881 (1985).

${ }^{34}$ R. Sizmann, J. Nucl. Mater. 69-70, 386 (1978).

${ }^{35} \mathrm{P}$. R. Bevington and D. K. Robinson, Data Analysis and Error Analysis for the Physical Sciences (McGraw Hill, New York, 1992), p. 102.

${ }^{36}$ Proceedings of the 6th International Discussion Meeting on Relaxations in Complex Systems, edited by K. L. Ngai and G. Ruocco [J. Non-Cryst. Solids 357, 241-782 (2011)].

${ }^{37}$ M. Atzmon and J. D. Ju, Phys. Rev. E 90, 042313 (2014).

${ }^{38}$ J. D. Ju and M. Atzmon, MRS Commun. 4, 63 (2014).

${ }^{39}$ H. B. Yu, W. H. Wang, H. Y. Bai, and K. Samwer, Natl. Sci. Rev. 1, 429 (2014).

${ }^{40}$ H. B. Yu, K. Samwer, Y. Wu, and W. H. Wang, Phys. Rev. Lett. 109, 095508 (2012).

${ }^{41}$ T. J. Lei and M. Atzmon, "Correlation between ductility of metallic glasses and their spectra of shear transformation zones" (unpublished). 